\title{
The associations between genetics, salt taste perception and salt intake in young adults
}

\author{
Leta Pilic and Yiannis Mavrommatis \\ St Mary's University, Twickenham, United Kingdom
}

\section{Abstract}

Hypertension is a major cause of cardiovascular disease and overall mortality. High dietary salt intake is one of the key risk factors for hypertension and in 2017, it was one of the three leading dietary risk factors for death and disability adjusted life years globally. Despite the efforts to change this behaviour, salt consumption still exceeds the recommendations. One of the main determinants of food intake, and potentially salt, is taste. Taste perception may be genetically determined, however research exploring the associations between genetics, salt taste perception and salt intake is scarce. This may be of special importance in younger adult populations where increased preference for salt is suggested. Therefore, the aim of this study was to explore the associations between genetics, salt taste perception (taste threshold and preference) and salt intake in young adults.

This study was approved by the St Mary's University Ethics Sub-Committee. Forty-two participants (18-35 years, 67\% female and $33 \%$ male) completed the study. Salt taste thresholds were identified using the British Standards Institution sensory analysis method (BS ISO 3972:2011) and preference for salty taste by asking participants how salty they usually prefer to eat their food using a Likert scale. Salt intake, expressed as mg sodium/1000 kcal, was measured using a five-step multiple pass 24 -hour recall for one day of the week and one weekend day. Participants were genotyped for two genetic variants in the $S C N N 1 B$ and $T R P V 1$ genes, which code for ion channels expressed in taste cells. Multiple regression analysis was performed including SCNN1B and TRPVI variants, salt taste threshold and preference as predictor variables and sodium intake $(\mathrm{mg} / 1000 \mathrm{kcal})$ as the dependent variable. Statistical significance was set at $\mathrm{p}<0.05$.

Participants were normal weight (Body Mass Index $23.8 \pm 3.7 \mathrm{~kg} / \mathrm{m}^{2}$ ), predominantly Caucasian with salt intake $7.5 \pm 2.7 \mathrm{~g}$ per day, reflecting current intakes in the UK. Regression model including genetics, thresholds and preference for salty taste explained $54 \%$ of the variance $(\mathrm{p}=0.028)$. In this model, TRPV1 variant rs8065080 $[\beta=422$, confidence interval $(50,794), p=0.030]$ and salt preference $[\beta=618$, confidence interval $(258,978), p=0.004]$ were indicated as predictors of sodium intake. These findings suggest that genetics and preference for salty taste may be drivers of salt intake in younger populations. If replicated, this information may in the future be used in designing more personalised approaches in changing this behaviour.

\section{Conflict of Interest}

Yiannis Mavrommatis is head of nutrigenetics at Nell Health Ltd. 